\title{
Crossover dynamics of dispersive shocks in Bose-Einstein condensates characterized by two- and three-body interactions
}

\author{
M. Crosta, ${ }^{1,2}$ S. Trillo, ${ }^{3}$ and A. Fratalocchi ${ }^{1, *}$ \\ ${ }^{1}$ PRIMALIGHT, Faculty of Electrical Engineering, Applied Mathematics and Computational Science, \\ King Abdullah University of Science and Technology (KAUST), Thuwal 23955-6900, Saudi Arabia \\ ${ }^{2}$ Dept. of Physics, Sapienza University of Rome, I-00185 Rome, Italy \\ ${ }^{3}$ Dipartimento di Ingegneria, Università di Ferrara, Via Saragat 1, I-44122 Ferrara, Italy
}

(Received 10 November 2011; published 10 April 2012)

\begin{abstract}
We show that the perturbative nonlinearity associated with three-atom interactions, competing with standard two-body repulsive interactions, can change dramatically the evolution of one-dimensional (1D) dispersive shock waves in a Bose-Einstein condensate. In particular, we prove the existence of a rich crossover dynamics, ranging from the formation of multiple shocks regularized by nonlinear oscillations culminating in coexisting dark and antidark matter waves to $1 \mathrm{D}$-soliton collapse. For a given scattering length, all these different regimes can be accessed by varying the density of atoms in the condensate.
\end{abstract}

DOI: 10.1103/PhysRevA.85.043607

PACS number(s): 03.75.Lm, 03.75.Kk, 42.65.Sf

\section{INTRODUCTION}

Bose-Einstein condensation (BEC) has been successfully described in the mean-field limit by the Gross-Pitaevskii equation (GPE), which embodies quantum two-body interactions (s-wave scattering) into its cubic nonlinear term [1]. Although not compulsory for (ideal, i.e., without interaction) condensation, such a term sustains several remarkable and otherwise unobservable coherent phenomena such as soliton formation [2], quantum shocks [3,4], collapse [5], nonclassical states [6], time reversal, and nonlinear Anderson localization [7]. The exceptionally huge tunability (in magnitude and sign) of the scattering length $a$ through Feshbach resonances [8] have made the area of ultracold atoms a prolific ground to investigate new quantum and nonlinear physics. Particular interest, in this context, was stirred by higher-order fewbody interactions [9], boosted also by the observation of resonant Efimov states [10]. High-order nonlinear terms can also arise from the crossover from three-dimensional (3D) to one-dimensional (1D) condensates as long as the transverse confinement settles in an strongly degenerate gas [11]. Most efforts in this area are presently aimed at assessing the impact of few-body recombination loss coefficients which limits the lifetime of condensates [12]. However, almost unexplored is also the effect of three-body elastic collisions, which naturally arises as a quintic conservative (Hamiltonian) term in the higher-order expansion of the GPE [9,13,14], whose impact clearly grows as higher densities are being reached in experiments [15]. It is therefore important to assess whether the three-body interactions can lead to a clear signature in terms of qualitative new scenarios, especially if the latter becomes accessible in the perturbative limit. In this paper we address this question with reference to the dynamics of dispersive shock waves (DSWs), which form in a repulsive BEC when the kinetic spreading regularizes the tendency driven by the nonlinearity to form multivalued wave fronts. At variance with earlier $[3,4,16]$ and more recent studies on DSWs [17-20], which are all based on the standard GPE, we

*www.primalight.org; andrea.fratalocchi@kaust.edu.sa investigate the case characterized by an attractive three-body nonlinear correction to the repulsive $s$-wave scattering. This regime is expected to be achievable by exploiting resonance tuning for bosons and turns out to be relevant also for superfluid fermions [21]. Unlike the case of competing nonlinearities of the same sign (where three-body contributions merely strengthen the nonlinearity at high densities), we find that the presence of the quintic term of different sign leads to a rich phase-diagram of the wave-breaking phenomenon, with novel regimes that uniquely identify the contribution of the threebody interaction. Remarkably, for a given scattering length, the crossover between different dynamics can be controlled by changing the atomic density (e.g., by changing the number of atoms) in the condensate. Due to the ubiquitous nature of DSWs, these findings are also of fundamental interest in other areas of classical physics, including nonlinear optics [22], electronic systems [23], and granular chains [24].

\section{MODEL AND GENERAL SHOCK SCENARIO}

We start from the following dimensionless cubic-quintic GPE, describing the free evolution in 1D (i.e., after releasing the trap along $x$ ) of a BEC ruled by two- and three-body interactions:

$$
i \epsilon \frac{\partial \psi}{\partial t}+\frac{\epsilon^{2}}{2} \frac{\partial^{2} \psi}{\partial x^{2}}-|\psi|^{2} \psi+\alpha|\psi|^{4} \psi=0 .
$$

In Eq. (1) $t, x$, and $\psi$ are linked to the corresponding realworld quantities $T, X$, and $\Psi$ as follows: $t=T \omega, x=X / X_{0}$, and $\psi=\Psi / \sqrt{\rho_{\infty}}$, where $\omega$ is the transverse BEC trap frequency, $X_{0}^{2}=g_{2} \rho_{\infty} /\left(m \omega^{2}\right)=4 \pi \rho_{\infty} a_{0}^{4} a\left(a_{0} \equiv \sqrt{\hbar /(m \omega)}\right.$ being the harmonic oscillator characteristic width, $m$ being the atomic mass), and $\rho_{\infty}$ is an asymptotic density which serves as a reference for the local density $|\Psi(X, T)|^{2}$. This normalization yields $\varepsilon=\left(a_{0} / X_{0}\right)^{2}=1 /\left(\rho_{\infty} a_{0}^{2} a\right)$, with $\varepsilon \ll 1$ in the regime considered here, while $\alpha=g_{3} \rho_{\infty} / g_{2}$ gives the relative strength of the three-body nonlinear coefficient $g_{3}$ [9] with respect to the two-body nonlinear coefficient $g_{2}=4 \pi \hbar^{2} a / m$. It is worth emphasizing that, at variance with the cubic GPE system $(\alpha=0)$ where the nonlinear response is completely characterized by the scattering length $a$, this 
normalization clearly shows that the impact of higher-order interactions grows for increasing densities (i.e., higher number of atoms in a given volume), and hence one has to deal with a nonlinear response that lives in a two-dimensional parameter space. This permits the exploration of different dynamical scenarios even for a constant scattering length $a$.

We study the general shock dynamics in the dispersionless (or hydrodynamic) limit of Eq. (1), which can be obtained by applying the Madelung transformation $\psi=\sqrt{\rho} \exp \left(\frac{i}{\epsilon} \int d x u\right)$ for $\epsilon \rightarrow 0$ :

$$
\frac{\partial \rho}{\partial t}+\frac{\partial(u \rho)}{\partial x}=0, \quad \frac{\partial u}{\partial t}+u \frac{\partial u}{\partial x}+\frac{\partial f}{\partial x}=0,
$$

where $f(\rho)=\rho-\alpha \rho^{2}$, and $\rho(x, t)$ and $u(x, t)$ act as density and velocity of the fluid, respectively. We study the impact of the quintic term by investigating the decay behavior of an initial jump in the density (i.e., the so-called Riemann problem of hydrodynamics). This problem is important not only because it is amenable to an explicit solution (unlike the Cauchy problem for a generic initial condition), but also because it is representative of the dynamics of smooth inputs which tends to develop a steep front (jump) in a finite time (gradient catastrophe), owing to a dominating nonlinearity which reflects the tendency of portions of the wave-packet with higher density to overtake those with lower density. Moreover, one could envisage an experiment where the abrupt jump in the density is suitably prepared at $t=0$, either removing a wall that divides two portions where atoms are condensed with different densities, or from a single condensate by exploiting the techniques discussed by Hoefer et al. [4], employing strong laser beams focused on the condensate. On the edge of the focused beam, the light pulse would push rapidly away the BEC atoms, thus realizing a sharp density variation in the condensate. We also point out that an ideal jump implies that the number of atoms $N$ (i.e., the integral of the density) diverges. However, this initial state is still representative of a real case (finite $N$ ) provided the density goes to zero over a spatial scale larger than that involved in the shock dynamics illustrated below.

Therefore, we start our theoretical analysis by solving Eqs. (2) with the initial value describing a density jump across $x=0: u(x, 0)=0, \rho(x, 0)=\rho_{0}^{+}+\left(\rho_{0}^{-}-\rho_{0}^{+}\right) \Theta(-x)[\Theta(x)$ is the Heaviside function]. Henceforth, we take without loss of generality a unit density $\left(\rho_{0}^{+}=1\right)$ for $x>0$ (this amounts to identifying the normalization constant $\rho_{\infty}$ as the asymptotic density $|\Psi|^{2}$ at $\left.x=+\infty\right)$ and denote the constant density in the semispace $x<0$ as $\rho_{0} \equiv \rho_{0}^{-}>1$. We solve Eqs. (2) by deriving the following Riemann invariants $\lambda_{ \pm}=u \pm R(\rho)$ :

$$
R(\rho)=\sqrt{\rho(1-2 \alpha \rho)}+\frac{\cos ^{-1}(1-4 \alpha \rho)}{2 \sqrt{2 \alpha}},
$$

which transform Eqs. (2) into the diagonal form

$$
\frac{\partial \lambda_{ \pm}}{\partial t}+V_{ \pm} \frac{\partial \lambda_{ \pm}}{\partial x}=0,
$$

where $V_{ \pm}=u \pm \sqrt{\rho(1-2 \alpha \rho)}$ are the Riemann characteristic eigenvelocities. As long as $V_{ \pm}$are strictly real, which requires

$$
\alpha<\frac{1}{2 \rho_{0}}, \quad \rho_{0} \equiv \rho_{0}^{-},
$$

the evolution of $\lambda_{ \pm}$can be given in terms of the self-similar variable $\zeta=x / t$ through the following simple wave solutions of the system (characterized by one of the two Riemann variables remaining constant):

$$
\begin{aligned}
& R\left(\rho_{0}^{-}\right)-R(\rho)-\sqrt{\rho(1-2 \alpha \rho)}=\zeta, \\
& R(\rho)-R\left(\rho_{0}^{+}\right)+\sqrt{\rho(1-2 \alpha \rho)}=\zeta .
\end{aligned}
$$

The two simple waves (6) are connected by an intermediate region with constant values $\rho=\rho_{i}$ and $u=u_{i}$. The latter can be found by the matching equations $u_{i}=\left[R\left(\rho_{0}^{+}=1\right)-\right.$ $\left.R\left(\rho_{0}\right)\right] / 2$ and $2 R\left(\rho_{i}\right)=R\left(\rho_{0}^{+}=1\right)+R\left(\rho_{0}\right)$. The borders $x_{i \pm}$ of the intermediate region are then calculated by substituting the density $\rho_{i}$ into Eqs. (6):

$$
\frac{x_{i \pm}}{t} \equiv \zeta_{i \pm}=R\left(\rho_{i}\right)-R\left(\rho_{0}\right) \pm \sqrt{\rho_{i}\left(1-2 \alpha \rho_{i}\right)} .
$$

Conversely, the simple wave external edges $x_{e \pm} \equiv \zeta_{e \pm} t$ are calculated by matching Eqs. (6) with the asymptotic (input) values $\rho_{0}^{+} \equiv 1$ and $\rho_{0}^{-} \equiv \rho_{0}$, thus obtaining

$$
\frac{x_{e-}}{t}=-\sqrt{\rho_{0}^{-}\left(1-2 \alpha \rho_{0}^{-}\right)}, \quad \frac{x_{e+}}{t}=\sqrt{1-2 \alpha} .
$$

Equations (6)-(8) predict a rich scenario for the shock dynamics, as illustrated in Figs. 1(a) and 1(b). For small values of $\alpha$ [see Fig. 1(a)], wave breaking occurs only for $x>0$, where the velocity $\zeta_{i+}$ of the point $x_{i+}$ is larger than $\zeta_{e+}$, leading to a multivalued region whose dispersive regularization yields a DSW (see below). However, a more complex dynamics is observed when $\alpha$ is increased [see Fig. 1(b)]: in this case

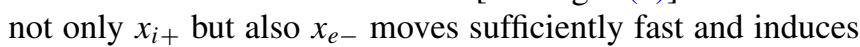
wave breaking for $x<0$, thus producing a double shock in the dynamics. The crossover between these two regimes is given by the condition of having an infinite steepness $\left[\partial \rho\left(\xi, \alpha_{c}\right) / \partial \xi \rightarrow \infty\right]$ when $\rho=\rho_{0}$, which is equivalent to

$$
\left.\frac{\partial \xi\left(\rho, \alpha_{c}\right)}{\partial \rho}\right|_{\rho_{0}}=0
$$

By using the first of Eqs. (6) we obtain, after straightforward algebra, the value of the critical $\alpha_{c}$ that yields the transition to
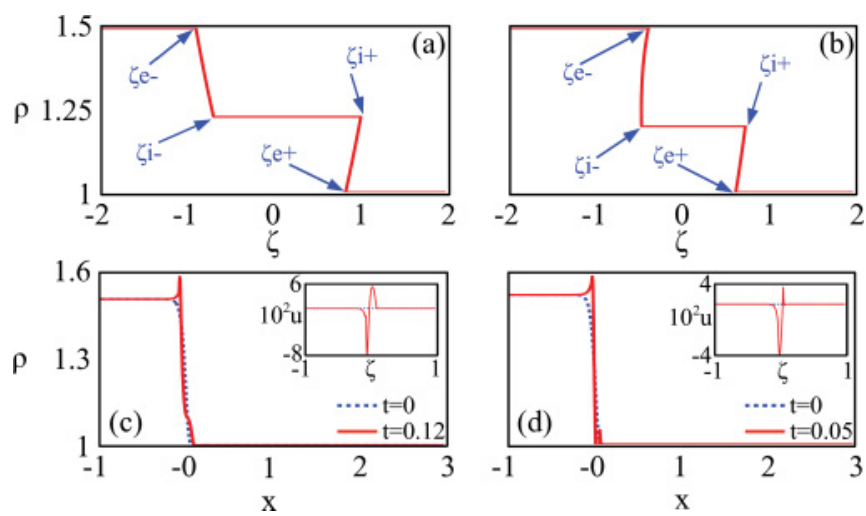

FIG. 1. (Color online) Dispersionless evolution for $\rho_{0}=1.5$ as obtained from Eqs. (5)-(7) with (a) $\alpha=0.15\left(\alpha<\alpha_{c}\right)$ and (b) $\alpha=0.3\left(\alpha>\alpha_{c}\right)$ Numerical results from Eqs. (2) comparing the case (c) $\alpha=0.4$ with the case (d) of a standard GPE ( $\alpha=0)$ with positive cubic nonlinearity (i.e., attractive interactions). 


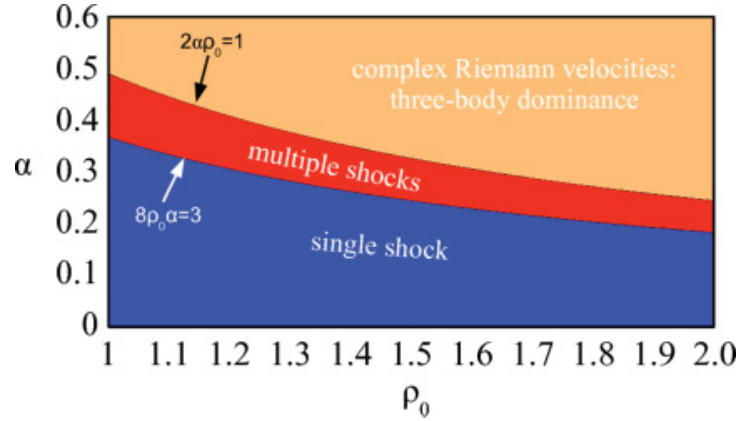

FIG. 2. (Color online) Global phase diagram of shock dynamics in parameter plane $\left(\rho_{0} \equiv \rho_{0}^{-}, \alpha\right)\left[\rho_{0}^{+}=1\right]$.

the double shock dynamics:

$$
\alpha_{c}=\frac{3}{8 \rho_{0}} .
$$

It is worth noting that a double shock has also been predicted in a BEC flowing through a penetrable barrier [18], although both the underlying mechanism and the regularization are completely different in the latter case.

A third regime settles in when Eq. (5) is violated, resulting in imaginary eigenvelocities $V_{ \pm}$[Eqs. (2) are no longer hyperbolic]. In this case, solutions (6)-(8) are meaningless and we resort to numerical integration of Eqs. (2). Our analysis shows [Fig. 1(c)] that the system evolution is characterized by the generation of two opposite velocities [Fig. 1(c), $u$ in the inset], which compress the wavefront and generate a cusp-like singularity at $x \approx 0^{-}$. This behavior originates from a three-body dominance over the two-body term, as demonstrated by comparing Fig. 1(c) to Fig. 1(d), which shows the evolution of the same input launched in a standard GPE with a positive (attractive) nonlinearity [which can be obtained by putting $\alpha=0$ in Eq. (1) and changing $x \rightarrow$ $i x$ in order to have $\left.\partial^{2} / \partial x^{2} \rightarrow-\partial^{2} / \partial x^{2}\right]$. The two wave breakings are qualitatively identical, which can be understood by considering that the GPE with an attractive two-body nonlinearity exhibits complex eigenvelocities, in the same way as Eq. (1) for $\alpha>1 / 2 \rho_{0}$. Despite such similarity, the long-term dynamics (regularization) in the presence of threebody nonlinearities will be totally different, as discussed in the following paragraphs. Figure 2 summarizes the results of the dispersionless analysis in a wave-breaking phase diagram. In particular, the two curves $\alpha^{*}=\alpha^{*}\left(\rho_{0}\right)$ and $\alpha=1 /\left(2 \rho_{0}\right)$ divide the phase space into three distinct regions, each characterized by the different wave-breaking scenarios deepened below.

\section{REAL EIGENVELOCITIES: TUNING SHOCK DYNAMICS}

We study the shock regularization for $\alpha \leqslant \alpha^{*}$ by exploiting the Whitham theory of modulation in the form suitable for nonintegrable systems [25]. We summarize only the outcome of this approach, while deferring the (involved) mathematical details to a future presentation. When $\alpha \leqslant \alpha^{*}$, the multivalued region for $x>0$ (Fig. 1) is regularized by the formation of a single DSW such that a modulated cnoidal wave appears within a shock fan limited by a leading $x_{l}$ and a trailing $x_{t}$ edge. By matching the dispersionless limit with the Whitham equations
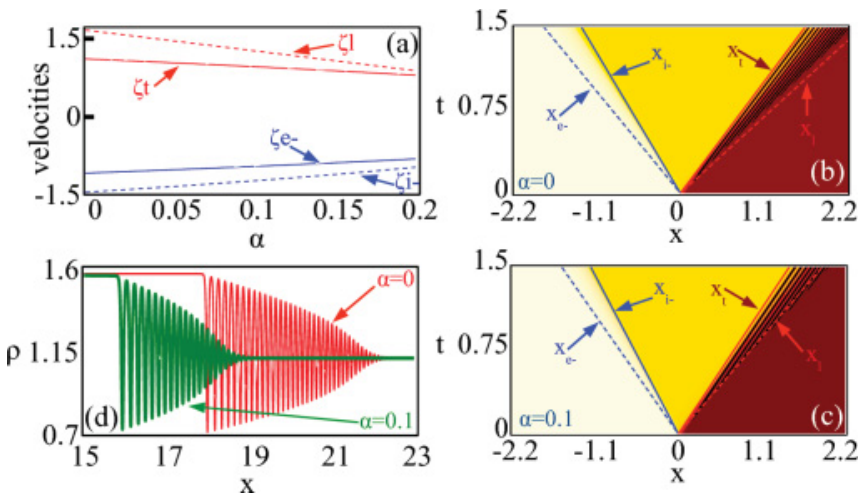

FIG. 3. (Color online) (a) Velocity of DSW edges $\zeta_{t}, \zeta_{l}$, and rarefaction edges $\zeta_{e-}, \zeta_{i-}$ vs $\alpha$; Color level plot of density evolution $\rho(x, t)$ from Eq. (1) for $\epsilon=0.01$ and (b) $\alpha=0$, (c) $\alpha=0.1$. For comparison, the edges of the DSW $x_{l}, x_{t}$ from Eq. (12), $x_{e-}, x_{i-}$ of the rarefaction wave from Eqs. (7) and (8) are reported. (d) Snapshots $\rho(x, t=8)$ from (b) and (c) showing the DSW modulated cnoidal wave for $\alpha=0,0.1$.

[25], we are able to find the velocities of the edges $\zeta_{l}=x_{l} / t$ and $\zeta_{t}=x_{t} / t$ (replacing the hydrodynamic estimates $\zeta_{e+}, \zeta_{i+}$ ) in the form

$$
\begin{aligned}
& \zeta_{l}=(1-2 \alpha)\left(2 \gamma_{l}-\frac{1}{\gamma_{l}}\right), \\
& \zeta_{t}=R\left(\rho_{i}\right)-R(1)+\gamma_{t} \sqrt{\rho_{i}\left(1-2 \alpha \rho_{i}\right)},
\end{aligned}
$$

with $\gamma_{l}=\gamma(1)$ and $\gamma_{t}=\gamma\left(\rho_{i}\right)$ arising from the solution of the differential equation

$$
\frac{\partial \gamma}{\partial \rho}=\frac{(1+\gamma)(1+2 \gamma-8 \gamma \alpha)}{2 \rho(2 \gamma+1)(2 \alpha \rho-1)},
$$

integrated over $\rho \in\left[1, \rho_{i}\right]$ with the initial condition $\gamma(1)=1$ for $\gamma_{l}$ and $\gamma\left(\rho_{i}\right)=1$ for $\gamma_{t}$. Figure 3(a) displays the DSW edge velocities $\zeta_{l}$ and $\zeta_{t}$ [calculated by integrating Eq. (12)] and those of the rarefaction wave $\zeta_{e-}$ and $\zeta_{i-}$ [from Eqs. (7) and (8)] as a function of $\alpha$. Increasing the three-body contributions results in a nearly linear decrease of all relevant velocities $\zeta_{l}$, $\zeta_{t}, \zeta_{e-}$, and $\zeta_{i-}$, with a consequent reduction of the extension of both simple waves and the shock fan. It is worth emphasizing that the quintic nonlinearity has a different impact on the two edges of the DSW, the leading edge $\zeta_{l}$ being more deeply

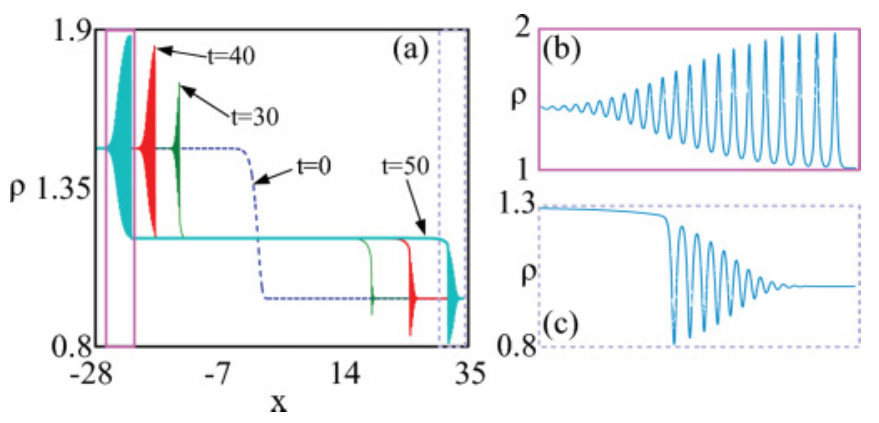

FIG. 4. (Color online) (a) Snapshots of density $\rho(x)$ obtained from Eq. (1) with $\alpha=0.3, \epsilon=0.01$, and a input jump $\rho_{0}=1.5$. Panels (b) and (c) present a zoom of the boxed evolution in (a), showing the opposite type of regularization taking place in the dynamics. 
affected than the trailing edge $\zeta_{t}$, as evident from the different slopes in Fig. 4(a) and the snapshots in Fig. 4(d). As a consequence, even when $\alpha$ is increased by a small factor, the shock dynamics is significantly affected in terms of both shock fan and overall angular shock direction. To verify these predictions, we resort to numerical integration of Eq. (1). As shown in Figs. 3(b) and 3(c), a remarkable agreement is obtained between theory and numerical simulations. The snapshot in Fig. 3(d) shows that, despite a small variation of $\alpha$, a substantial reduction of the shock fan extension occurs (nearly a factor of two for $\Delta \alpha=0.1$ ) accompanied by a reduced average velocity (center of mass closer to $x=0$ ). In summary, in the regime where a single shock is formed for $x>0$, all the features of the shock dynamics are significantly varied by slightly perturbing the strength of the three-body-interaction terms $\alpha$.

\section{REAL EIGENVELOCITIES: MULTISHOCK GENERATION THROUGH ANTIDARK SOLITONS}

When $\alpha^{*}<\alpha<1 / 2 \rho_{0}$, simulations show the occurrence of a second DSW for $x<0$ (Fig. 4), in complete agreement with the hydrodynamic analysis. In this case, however, the DSW for $x<0$ differs substantially from the dynamics discussed above for $x>0$. The latter, in fact, is characterized by a cnoidal wave composed by a train of dark-like oscillations (see Fig. 3), owing to the condition $\rho\left(x_{t}\right)>\rho\left(x_{l}\right)$ [see also Fig. 1(a)]. The DSW, more specifically, originates from a series of oscillations that start from the leading edge $x_{l}$ (where the shock matches the linear wave background at $\rho=1$ ) and culminate in a dark soliton at the trailing edge [Fig. 4(c)]. On the contrary, the DSW for $x<0$ moves in the opposite (backward) direction and has a larger density on the leading edge, which yields $\rho\left(x_{l 1}\right)>\rho\left(x_{t 1}\right)$, with $x_{l 1}$ and $x_{t 1}$ being the leading and trailing edge of the DSW at $x<0$, respectively. As a consequence, the modulated wave train that regularizes the shock needs to be composed by bright entities, as shown in Fig. 4(b). In this case, the trailing edge is an antidark (bright on pedestal) soliton solution of Eq. (1), which has been thoroughly investigated recently [26]. Quite remarkably, although these solitons are mostly unstable [26], the average velocity $v^{*}$ of the DSW for $x<0$ is such that the soliton (and hence the DSW) is totally stable on propagation. This can be rigorously demonstrated from the stability diagram of the antidark solitons of the cubicquintic nonlinear Schrödinger (CQNLS) equation (Fig. 5). The

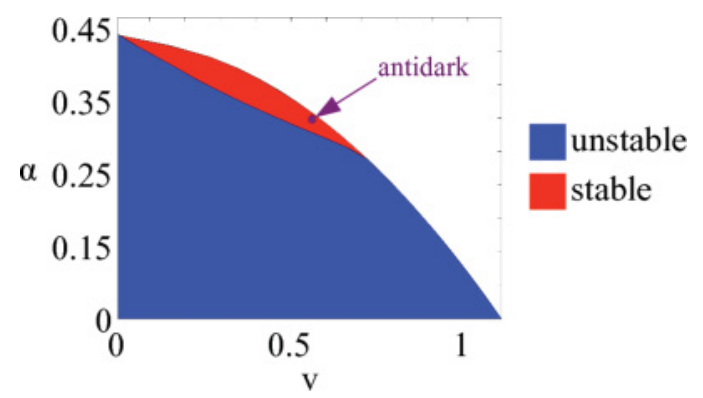

FIG. 5. (Color online) $(\alpha, v)$ Stability map of the antidark soliton solutions of the CQNLS for $\rho_{0}^{-}=1.5$, with $v$ being the soliton velocity in the $(t, x)$ plane. The position of the antidark soliton of Fig. 4(b) is indicated as a point in the figure.
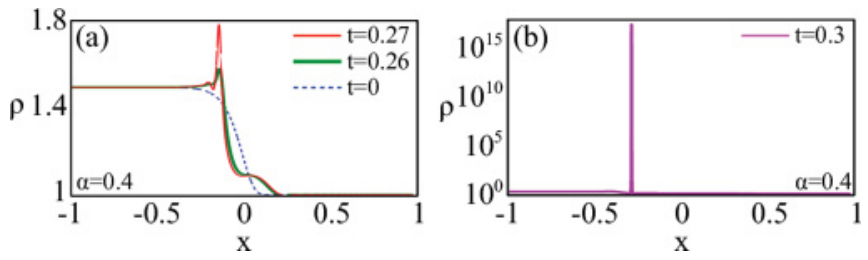

FIG. 6. (Color online) Snapshots of density $\rho$ vs $x$ from Eq. (1) for $\alpha=0.4$ and $\epsilon=0.01$ : (a) shock regularization, (b) antidark collapse instability.

stability map is constructed by following the analysis of Ref. [26]. As seen, the numerically calculated velocity $v^{*}$ of the antidark soliton of Fig. 4(b) lies in the stable region (Fig. 5, point), and we therefore observe a stable dynamical evolution of the DSW for $x<0$.

\section{COMPLEX EIGENVELOCITIES: 1D BEC COLLAPSE}

When $\alpha>1 / 2 \rho_{0}$, the wave breaking observed in the simulations of Eq. (1) reflects indeed the dominant character of three-body interactions [Figs. 6(a) and 6(b)]. In the early stage, a cusp-like singularity in $x \sim 0^{-}$is generated [Fig. 6(a)], as predicted by Eqs. (2). Contrary to the the case of the standard attractive GPE, which shows a similar early-stage behavior [see Fig. 1(d)], the three-body dominance in Eq. (1) leads to a completely different long-term dynamics. In the quintic case, in fact, the singularity tends to evolve into antidark solitons due to the condition $\rho\left(x_{l}\right)>\rho\left(x_{t}\right)$. Contrary to the previous case, however, here the solitons' velocity is nearly vanishing. Zero-velocity antidark solitons of Eq. (1) are always unstable [26], and they lead the BEC toward irreversible collapse near $x=0$ [Fig. 6(b)]. We emphasize that such a collapsing dynamics, which we have verified to occur also for different inputs (e.g., Gaussian on pedestal), is a unique features of Eq. (1). Collapse, in fact, cannot be observed in the standard GPE ( $\alpha=0$ ) that, owing to its integrable nature, does not possess unstable solitons. It is also worthwhile remarking the perturbative value of the quintic term $(\alpha>0.34)$ which induces such a dramatic and abrupt change in the dynamics.

\section{CONCLUSIONS}

In conclusion, we have shown that the perturbative nonlinearity arising from three-body elastic collisions can dramatically alter the breaking scenario in repulsive BEC. The generation of multiple DSWs involving antidark solitons as well as 1D collapsing dynamics are new peculiar behaviors that can be controlled by means of the number of BEC atoms. These results are expected not only to foster new perspectives in BEC physics, but also to stimulate novel experiments in nonlinear optics, where the quintic terms account for saturation of classical Kerr nonlinearities.

\section{ACKNOWLEDGMENT}

We acknowledge funding from PRIN 2009 project (No. 2009P3K72Z). 
[1] F. Dalfovo, S. Giorgini, L. V. Pitaevskii, and S. Stringari, Rev. Mod. Phys. 71, 463 (1999).

[2] C. Becker et al., Nat. Phys. 4, 496 (2008).

[3] Z. Dutton, M. Budde, C. Slowe, and L. V. Hau, Science 293, 663 (2001).

[4] M. A. Hoefer, M. J. Ablowitz, I. Coddington, E. A. Cornell, P. Engels, and V. Schweikhard, Phys. Rev. A 74, 023623 (2006).

[5] J. M. Gerton, D. Strekalov, I. Prodan, and R. G. Hulet, Nature (London) 408, 692 (2000).

[6] N. Bar-Gill, D. D. Bhaktavatsala Rao, and G. Kurizki, Phys. Rev. Lett. 107, 010404 (2011).

[7] J. Martin, B. Georgeot, and D. L. Shepelyansky, Phys. Rev. Lett. 101, 074102 (2008).

[8] C. Chin, R. Grimm, P. Julienne, and E. Tiesinga, Rev. Mod. Phys. 82, 1225 (2010).

[9] T. Köhler, Phys. Rev. Lett. 89, 210404 (2002); E. Braaten and H.-W. Hammer, Phys. Rep. 428, 259 (2006).

[10] T. Kraemer et al., Nature (London) 440, 315 (2006).

[11] F. Gerbier, Europhys. Lett. 66, 771 (2004).

[12] P. A. Altin, G. R. Dennis, G. D. McDonald, D. Doring, J. E. Debs, J. D. Close, C. M. Savage, and N. P. Robins, Phys. Rev. A 84, 033632 (2011).

[13] F. Kh. Abdullaev, A. Gammal, L. Tomio, and T. Frederico, Phys. Rev. A 63, 043604 (2001); F. Kh. Abdullaev and M. Salerno, ibid. 72, 033617 (2005).

[14] E. Reyes-Gomez, A. Bruno-Alfonso, S. B. Cavalcanti, and L. E. Oliveira, Phys. Rev. E 84, 036604 (2011).
[15] T. Shobu, H. Yamaoka, H. Imai, A. Morinaga, and M. Yamashita, Phys. Rev. A 84, 033626 (2011).

[16] A. M. Kamchatnov, R. A. Kraenkel, and B. A. Umarov, Phys. Rev. E 66, 036609 (2002); B. Damski, Phys. Rev. A 69, 043610 (2004); V. M. Perez-Garcia, V. V. Konotop, and V. A. Brazhnyi, Phys. Rev. Lett. 92, 220403 (2004).

[17] J. J. Chang, P. Engels, and M. A. Hoefer, Phys. Rev. Lett. 101, 170404 (2008).

[18] A. M. Leszczyszyn, G. A. El, Yu. G. Gladush, and A. M. Kamchatnov, Phys. Rev. A 79, 063608 (2009).

[19] R. Meppelink, S. B. Koller, J. M. Vogels, P. van der Straten, E. D. van Ooijen, N. R. Heckenberg, H. Rubinsztein-Dunlop, S. A. Haine, and M. J. Davis, Phys. Rev. A 80, 043606 (2009).

[20] G. Dekel, V. Farberovich, V. Fleurov, and A. Soffer, Phys. Rev. A 81, 063638 (2010).

[21] P. Pieri and G. C. Strinati, Phys. Rev. Lett. 91, 030401 (2003).

[22] J. E. Rothenberg and D. Grischkowsky, Phys. Rev. Lett. 62, 531 (1989); S. Jia, W. Wan, and J. W. Fleischer, ibid. 99, 223901 (2007); C. Conti, A. Fratalocchi, M. Peccianti, G. Ruocco, and S. Trillo, ibid. 102, 083902 (2009).

[23] E. Bettelheim, A. G. Abanov, and P. Wiegmann, Phys. Rev. Lett. 97, 246401 (2006).

[24] A. Molinari and C. Daraio, Phys. Rev. E 80, 056602 (2009).

[25] G. A. El, Chaos 15, 037103 (2005).

[26] M. Crosta, A. Fratalocchi, and S. Trillo, Phys. Rev. A 84, 063809 (2011). 